\title{
Influencia del software GeoGebra en el aprendizaje de la geometría en estudiantes de cuarto grado de secundaria en el distrito de Tambopata de la región de Madre de Dios
}

GeoGebra software influence on learning geometry in fourth grade high school students in the Tambopata district of the Madre de Dios region

\section{Eliseo Pumacallahui Salcedo ${ }^{1}$ \\ Cory Ingrid Acuña Quispe ${ }^{2}$ \\ Dominga Asunción Calcina Álvarez ${ }^{3}$}

\begin{abstract}
Resumen: El objetivo de la investigación que se reporta en esta comunicación ha sido determinar la influencia del software GeoGebra en el aprendizaje de la geometría en estudiantes de cuarto grado de educación secundaria de la institución educativa Señor de los Milagros. El problema que motivó la investigación y una intervención didáctica fue el bajo nivel de aprendizaje de los estudiantes en los cursos de matemática, en especial en geometría. El estudio es cuasi experimental con una muestra de 67 estudiantes, distribuidos en dos grupos, que fueron evaluados con una prueba de evaluación del aprendizaje en geometría aplicada después de la intervención. Los resultados muestran que la intervención didáctica basada en el empleo del software GeoGebra, mejoró significativamente
\end{abstract}

Fecha de recepción: 08 de julio de 2020. Fecha de aceptación: 01 de julio de 2021.

${ }^{1}$ Universidad Nacional Intercultural de Quillabamba, Cusco, Perú, eliseo.pumacallahui@uniq.edu.pe, https://orcid.org/0000-0002-5730-684X

2 Seguro Social de Salud de Quillabamba, Cusco, Perú. coryquispe@hotmail.com , https://orcid.org/00000003-3450-7187

3 Universidad Nacional Amazónica de Madre de Dios, Perú. dcalcina@unamad.edu.pe, https://orcid. org/0000-0002-6869-0939 
el aprendizaje de la geometría en estudiantes de cuarto grado de secundaria. Los estudiantes del grupo experimental y del grupo de control obtuvieron un promedio de 13.3611 y 8.9354 puntos respectivamente, dando una diferencia de 4.4257 puntos a favor del grupo experimental.

Palabras claves: GeoGebra, aprendizaje, estudiantes, grupo experimental, grupo control.

\begin{abstract}
The objective of this research has been to determine the influence of GeoGebra software on the learning of geometry in fourth grade students of secondary education at the Señor de los Milagros educational institution. The research problem was the presence of the low level of student learning in mathematics courses, especially in geometry. The study is quasi-experimental and the sample was 67 students divided into two groups. Both groups were evaluated with a geometry learning assessment test that was applied after the intervention. The results were finally discussed. The use of GeoGebra software significantly improved the learning of geometry in fourth grade high school students. Where the students of the experimental and control group obtained an average score of 13.3611 and 8.9354 points respectively, giving the difference of 4.4257 points in favor of the experimental group.
\end{abstract}

Keywords: GeoGebra, learning, students, experimental group, control group.

\title{
INTRODUCCIÓN
}

La investigación aborda la influencia del software GeoGebra en el diseño de una intervención didáctica para mejorar el aprendizaje de la geometría; específicamente, en las temáticas de triángulos, cuadriláteros y la circunferencia, en estudiantes del cuarto grado de secundaria. La motivación para desarrollar la investigación fue la evidencia el problema del bajo nivel de aprendizaje de los estudiantes en los cursos de matemáticas.

En el año 2018, el Perú se ubicó en el puesto 64 de 77 países en la prueba de lógico matemático, los datos provienen del Programa para la Evaluación Internacional de Estudiantes (PISA, 2018) y de la Organización para la Cooperación y el Desarrollo Económico (OCDE), estos resultados de las evaluaciones 
internacionales indican que en los cursos de matemáticas el sistema educativo peruano necesita mejorar las estrategias de enseñanza (Lamana Selva y De La Peña, 2018), el problema también se extiende a los estudiantes universitarios, en especial en los primeros semestres académicos, ocasionando altas tasas de repitencia, abandono de estudios universitarios, inserción al pandillaje y limitaciones para continuar los estudios universitarios.

El sistema educativo peruano requiere plantear exigencias y alternativas de solución referentes a las estrategias dinámicas y de laboratorio para compartir experiencias y conocimientos en el área de la matemática, básicamente la geometría. Por otro lado, para mejorar la calidad de la educación básica regular y sus resultados no es suficiente aplicar pruebas y darle coherencia al currículo, sino reorientar las prácticas pedagógicas con el uso de metodologías innovadoras con apoyo de programas computacionales que permiten innovar el proceso de enseñanza-aprendizaje, evitando el tradicional paradigma de copiar página y repetir sin relacionar el conocimiento; así como disminuir la deficiente formación profesional docente (Consejo Nacional de Educación, 2006).

Las tecnologías de información y comunicación (TIC) en el ámbito educativo han generado nuevas maneras de pensar y regir los procesos de aprendizaje y enseñanza. Con la llegada de estas tecnologías se han sentado diversas posturas en cuanto a su incorporación e integración curricular y a las ventajas o desventajas que podrían tener en el desempeño académico de los estudiantes. La matemática es una de las áreas de la ciencia en donde la tecnología ha avanzado significativamente, con la creación de diferentes programas computacionales matemáticos crecen las expectativas por diseñar e implementar objetos y ambientes de aprendizaje que promuevan una mejor comprensión de conceptos matemáticos y que al mismo tiempo sirvan de apoyo al trabajo en clase y motiven a los estudiantes al estudio independiente (Córdoba et al..,2013).

Al respecto, Marqués (1995), citado por Cárdenas (2018, p. 10), explica que el software matemático, "constituye un estupendo laboratorio matemático que permite experimentar, suplir carencias en el bagaje matemático del estudiante, desarrollar la intuición, conjeturar, comprobar, demostrar, y, en definitiva, ver las situaciones matemáticas de una forma práctica". Sin embargo, los docentes del área de matemática, en especial de la geometría siguen insistiendo en la enseñanza caracterizada por la memorización de conceptos, propiedades, fórmulas y teoremas; privilegiando el uso de recursos como la pizarra, el plumón, la escuadra y el transportador hechos de madera; dejando de lado recursos tecnológicos, debido probablemente al desconocimiento de su empleo y su 
potencial para dinamizar el trabajo en clase. Lo que puede tener efectos en el aprendizaje. Al respecto Cárdenas (2018) señala que los modelos tradicionales han afectado a muchos estudiantes, porque constituye el punto de quiebre para crear en ellos desidia, rechazo y resistencia hacia la matemática debido al cansancio que les genera realizar ejercicios rutinarios, mientras que las actividades basadas en las nuevas tecnologías son más dinámicas y divertidas. Por ello, se señala que el empleo adecuado de un software educativo sirve de apoyo al proceso de enseñanza aprendizaje.

El interés de este trabajo es generar una estrategia de enseñanza dinámica con el empleo del software GeoGebra, que puede ser aplicable a todo nivel educativo, considerado como una herramienta didáctica utilizada por quien enseña y quien aprende, lo que permite al usuario alcanzar determinados propósitos (Gross-Salvat, 2000, p. 213). El software matemático GeoGebra es un elemento físico o simbólico que, dentro del aula de clase, provee de cierta ventaja al maestro para la presentación de una temática en particular, que le proporcione al estudiante una forma de representación, visualización y organización de los conceptos trabajados en el estudio de la geometría. (Falen, 2017, p. 35).

Según el Programa Curricular de Educación Secundaria del Ministerio de Educación (MINEDU) del Perú (2017) la competencia "Resuelve problemas de forma, movimiento y localización", "consiste en que el estudiante se oriente y describa la posición y el movimiento de objetos y de sí mismo en el espacio, visualizando, interpretando y relacionado las características de los objetos con formas geométricas bidimensionales y tridimensionales; implica que realice mediciones directas o indirectas de la superficie, del perímetro, del volumen y de la capacidad de los objetos, planos maquetas, usando instrumentos, estrategias y procedimientos de construcción y medida" (p. 147).

Asimismo, el Programa Curricular de Educación Secundaria del Ministerio de Educación (MINEDU) del Perú (2017) señala que la competencia transversal "Se

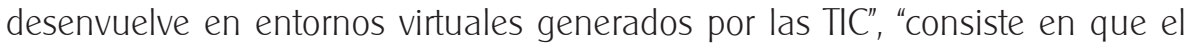
estudiante interprete, modifique y optimice entornos virtuales durante el desarrollo de actividades de aprendizaje y en prácticas sociales. Esto involucra la articulación de los procesos de búsqueda, selección y evaluación de información; de modificación y creación de materiales digitales, de comunicación y participación en comunidades virtuales, así como la adaptación de los entornos virtuales de acuerdo a sus necesidades e intereses de manera sistemática" (p. 215).

Por las consideraciones anteriores, el objetivo de este trabajo de investigación subyace en determinar la influencia del uso del software GeoGebra en el 
aprendizaje de la geometría en las temáticas de triángulos, cuadriláteros y la circunferencia en estudiantes de cuarto grado de educación secundaria de la institución educativa Señor de los Milagros del distrito y provincia de Tambopata de la región de Madre de Dios. La pregunta central de la investigación es: ¿En qué medida un diseño didáctico fundamentado en el uso del software GeoGebra influye el aprendizaje de la geometría en estudiantes del cuarto grado de educación secundaria?

La enseñanza de matemática en todos los niveles de educación, especialmente en educación secundaria, es un producto cultural dinámico, es cambiante, en constante desarrollo y reajuste. Al plantear y resolver problemas, Ios estudiantes se enfrentan a retos para los cuales no conocen de antemano las estrategias de solución; esta situación implica desafíos que van en busca de estrategias y recursos que activen la enseñanza de la matemática desde una perspectiva dinámica. El trabajo de investigación plantea que la estrategia didáctica fundamentada en el empleo del software GeoGebra, puede constituir una propuesta para mejorar la calidad de enseñanza de la geometría en estudiantes del cuarto grado de educación secundaria. Asimismo, se pretende optimizar el uso de los entornos proporcionados por las TIC que permitan la interacción entre los estudiantes y el docente.

El aporte al estado de conocimiento de esta investigación es incorporar al proceso de enseñanza de la geometría, el empleo del software GeoGebra brindando al docente de matemática un recurso didáctico tecnológico, para abordar de manera dinámica, pero con el rigor matemático, la enseñanza de la geometría, reemplazando así la enseñanza tradicional basada en la pizarra física y plumón que suelen conducir a una forma mecánica de plantear los temas de triángulos, cuadriláteros y circunferencias. Y al proceso de aprendizaje, porque le permite al estudiante, interactuar con los objetos y sus relaciones geométricas para adquirir conocimientos y aplicarlos a la resolución de problemas de la geometría.

\section{MARCO TEÓRICO}

\section{ANTECEDENTES DEL TRABAJO}

Diaz-Nunja et al. (2018), realizaron un estudio sobre los efectos del empleo del software GeoGebra en la enseñanza de la geometría con estudiantes de educación 
secundaria, enfocado en el desarrollo de capacidades de la matemática de educación básica regular: razonamiento y demostración, la comunicación matemática y la resolución de problemas. Se consideraron dos grupos, el experimental con el empleo del software GeoGebra y el grupo de control expuesto a una enseñanza tradicional sin el empleo del software. Ambos grupos fueron evaluados con una prueba del aprendizaje en geometría que se aplicó en momentos antes y después de la intervención. Los resultados sugieren que el empleo del software GeoGebra tuvo efectos en el fortalecimiento de las tres capacidades, con mejoras que resultaron significativas a niveles altos. También que las puntuaciones alcanzadas en el momento después fueron favorables al grupo intervenido en las tres capacidades, con diferencias significativas a niveles moderados.

Ruiz-López (2012), analizó el desarrollo de competencias geométricas y didácticas mediante el software GeoGebra en la formación inicial del profesorado de primaria. Trabajó con un grupo experimental, que había seguido el mismo proceso formativo que el grupo control, pero añadiendo el entorno GeoGebra para la resolución de problemas geométricos. Los resultados fueron estadísticamente significativos en la mejora de competencias didáctico-geométricas, a pesar de haber utilizado como instrumento de medida una prueba de lápiz y papel. Por su parte, Pablo (2016), realizó una investigación con el propósito de analizar si la aplicación del software GeoGebra influye significativamente en el aprendizaje de la geometría analítica en los estudiantes de quinto grado de educación secundaria. Participaron 60 estudiantes, los grupos experimental y de control estuvieron conformados por 30 estudiantes cada uno. Los resultados estadísticos indican que en el post-test efectuado, el grupo experimental obtiene un mayor desempeño que el grupo de control. Estos tres estudios sugieren que tanto los estudiantes como los futuros docentes, mejoraron su desempeño al utilizar el software GeoGebra. Un análisis sobre las tareas propuestas permitiría analizar más profundamente el tipo de actividad generada por los participantes y el empleo de GeoGebra.

\section{El SOFTWARE GEOGEBRA}

GeoGebra es un software libre, y puede considerarse como un software de geometría dinámica, pero que incluye otras particularidades algebraicas y de cálculo que permiten relacionar varias áreas matemáticas (Ruiz-Lopez, 2012). En nuestro caso hemos empleado el software GeoGebra fundamentalmente para resolver problemas de geometría, en especial en las temáticas de triángulos, 
cuadriláteros y de las propiedades de la circunferencia. La idea básica de los creadores y desarrolladores de este software (Hohenwarter, 2002; Hohenwarter et al.., 2009), ha sido unir geometría, álgebra y cálculo (las distintas representaciones de un mismo objeto se conectan dinámicamente) en un único programa de uso intuitivo que permita la enseñanza de las matemáticas en todos los niveles educativos, citado por (Ruiz-López, 2012, p. 55). Diaz-Nunja, et al. (2018) señalan que "la enseñanza de la geometría escolar desde una perspectiva dinámica es un campo relativamente nuevo en la docencia, pero con una presencia cada vez más frecuente y relevante (Ferreira, et al., 2009; Duval, 2000). Por ello, el National Council of Teachers of Mathematics (NCTM) promueve el uso de software de geometría dinámica en las aulas, entre ellos GeoGebra" (pp. 221-222). Estos mismos autores señalan atributos y características de Geogebra, mediante la tabla que se presenta a continuación.

Tabla 1. Atributos y características del software GeoGebra

\begin{tabular}{ll}
\hline \multicolumn{1}{c}{ Atributos } & \multicolumn{1}{c}{ Características } \\
\hline Constructividad & $\begin{array}{l}\text { Posibilidad de construir nuevos escenarios a partir de la combina- } \\
\text { ción de objetos en espacio y tiempo. Concepto estrechamente vincu- } \\
\text { lado al modelo constructivista del aprendizaje. } \\
\text { Navegabilidad }\end{array}$ \\
& $\begin{array}{l}\text { Posibilidad de explorar de manera libre y flexible, a diferencia de } \\
\text { otros paquetes que emplean rutas fijas, lineales y secuenciales. }\end{array}$ \\
Interactividad & Sistema que provee al usuario retro-alimentación en tiempo real, \\
& además de adaptar o modificar dinámicamente su comportamiento \\
en función de los eventos e información recibida. & \\
& Fiabilidad, relevancia, organización y accesibilidad de la información \\
que contiene el software, que adicionalmente puede ser adaptada a & diversos tipos de audiencias \\
Interfaz & Pantalla con que el aprendiz interactúa, que captura la atención, \\
& guía sus acciones y refleja el estado del sistema \\
\hline
\end{tabular}

Fuente: (Diaz-Nunja, et al., 2018, p. 222).

Otros estudios que se hicieron en el marco de la capacitación de docentes con el empleo de GeoGebra reportan que los docentes tuvieron opiniones favorables a su empleo en la enseñanza, si se tiene como base una capacitación adecuada (Tatar y Yilmaz, 2016; Bulut y Bulut, 2011). Todo ello, requiere de ser analizado en el contexto regional educativo peruano, que permita analizar la forma en que los 
estudiantes emplean GeoGebra y lo que su actividad puede generar en la visión de los profesores. Asimismo, la forma en que puede sensibilizarse a las autoridades educativas para promover las condiciones materiales, que posibiliten su uso, su análisis y su incorporación en el corto, mediano y largo plaza en la enseñanza regular.

\section{APRENDIZAJE DE LA GEOMETRÍA}

La importancia de la geometría como una materia del currículo escolar ha sido ampliamente reconocida por autores como Almeida (2002), citado por Gamboa y Ballesteros, (2009, p. 114), "quien señala que existen algunos objetivos generales que todo ciudadano debería alcanzar durante su formación básica: tener una cultura geométrica con visión histórica e interdisciplinaria, aplicar conocimientos geométricos para modelar en el campo científico, crear o resolver problemas reales, usar los diferentes lenguajes y representaciones, entre otros". De acuerdo con Duval (2000), citado por Diaz-Nunja, et al. (2018), "el aprendizaje de la geometría involucra, como mínimo, realizar tres actividades cognitivas: la construcción, que alude al diseño de configuraciones mediado por instrumentos geométricos; el razonamiento, vinculado con los procesos discursivos y de argumentación; y la visualización, que se enfoca en las representaciones espaciales" (p. 223).

Por otra parte, el Programa Curricular de Educación Secundaria (2016) del Ministerio de Educación (MINEDU) del Perú, indica, "que el aprendizaje de la matemática contribuye a formar ciudadanos capaces de buscar, organizar, sistematizar y analizar información para entender e interpretar el mundo que los rodea, desenvolverse en él, tomar decisiones pertinentes, y resolver problemas en distintas situaciones usando, de manera flexible, estrategias y conocimiento matemáticos" (MINEDU, 2016, p. 147). Además, el logro del perfil egresado de los estudiantes de Educación Secundaria requiere el desarrollo de cuatro competencias en el "área de matemática", a través del enfoque centrado en la resolución de problemas, como lo es la competencia "Resuelve problemas de forma, movimiento y localización", detallada anteriormente. Asimismo, dentro de los "estándares de aprendizaje" de dicha competencia, en el nivel esperado V, el estudiante resuelve problemas en los que modela las características y la ubicación de objetos a formas bidimensionales y tridimensionales, sus propiedades, su aplicación, reducción o rotación. Describe y clasifica prismas rectos, cuadriláteros, triángulos, círculos, por sus elementos: vértices, lados, caras, ángulos, y por sus propiedades, usando el lenguaje geométrico. Realiza giros en cuartos y 
medias vueltas, traslaciones, ampliación y reducción de formas bidimensionales en el plano cartesiano. Emplea procedimientos e instrumentos para ampliar, reducir, girar y construir formas; así como para estimar o medir la longitud, superficie y capacidad de los objetos.

\section{PROGRAMA DE INTERVENCIÓN}

En el marco de la estrategia de intervención del aprendizaje se elaboraron dos guías de aprendizaje, una para el grupo experimental y otra para el grupo de control. Se siguieron los objetivos del trabajo de investigación y los contenidos del Programa Curricular de Educación Secundaria del Ministerio de Educación del Perú, en las temáticas de triángulos, cuadriláteros y circunferencias.

La guía de aprendizaje para el grupo experimental estuvo apoyada para la enseñanza de una geometría dinámica con el uso del software GeoGebra. Tuvo la siguiente estructura: introducción para el estudiante, objetivos de aprendizaje, marco teórico, instrucciones para utilizar el software GeoGebra, actividades de aprendizaje y actividades de evaluación. La guía de aprendizaje para el grupo de control, incluía introducción para el estudiante, objetivos del aprendizaje, marco teórico, actividades de aprendizaje y actividades de evaluación; excepto las instrucciones para utilizar el software GeoGebra. Es decir, los contenidos y temáticas de la geometría, basada en la enseñanza de la geometría tradicional sin el uso de ninguna herramienta tecnológica.

El propósito del programa de intervención fue fortalecer el aprendizaje de la geometría con el empleo del software GeoGebra en las temáticas de triángulos, cuadriláteros y la circunferencia en comparación con la enseñanza tradicional. Se trabajó con un enfoque por competencias, centrado en la resolución de problemas. Ambas orientaciones pedagógicas son parte de las recomendaciones dadas por el MINEDU como parte del Programa Curricular de Educación Secundaria (2016) en el área de matemática.

El programa de intervención estuvo compuesto de 12 sesiones de aprendizaje por dos horas cada una y por el mismo docente del curso de geometría. La sesión del aprendizaje para el grupo experimental se aplicó en la sala de cómputo de la institución educativa, cada estudiante contó con un equipo de cómputo. Por su parte, la sesión del aprendizaje para el grupo de control se realizó en las aulas de clases con las mismas temáticas y ejercicios 
del grupo experimental, sin utilizar el software GeoGebra y por el mismo docente. La intervención en total abarcó dos meses.

En las sesiones de clase los estudiantes del grupo experimental mediante el software GeoGebra construyeron triángulos, cuadriláteros y circunferencias y aprendieron cómo medir los ángulos, lados, el área y el perímetro de dichas figuras geométricas; luego se formalizaron las definiciones, propiedades y teoremas relacionados a triángulos, cuadriláteros y de la circunferencia para dar respuestas a situaciones problemáticas, mientras los estudiantes del grupo de control utilizaron directo las propiedades y teoremas relacionados a triángulos, cuadriláteros y de la circunferencia. En general, en las 12 sesiones se puso énfasis en el empleo de metodologías y didácticas basadas en la problematización. El docente y los estudiantes trabajaron apoyándose en las guías de aprendizaje. Cada estudiante contó con una de estas guías, a partir de las cuales el docente les acompañaba en la identificación de carencias o contradicciones, con el propósito de facilitar la solución de problemas. En términos operativos, el docente ofrecía a estudiantes situaciones problemáticas de las guías para que construyeran y descubrieran las propiedades y teoremas con el apoyo del software GeoGebra.

\section{MATERIALES Y MÉTODOS}

El tipo de investigación es aplicada, porque se trata de un estudio de aula, enfocado en analizar el quehacer del maestro, que debe ser de permanente búsqueda de nuevas tecnologías y la adaptación y aplicación de nuevas teorías a la práctica de la educación, a la pedagogía experimental, con la finalidad de transformar la realidad educativa. Específicamente, en este caso se pretende analizar la eficacia de la variable independiente, es decir, el empleo del software GeoGebra como sustento de una estrategia de aprendizaje desarrollada por un mismo docente en el grupo experimental y en el grupo de control.

\section{DISEÑNO DE LA INVESTIGACIÓN}

El diseño de investigación es cuasi-experimental ya que pretende determinar la influencia del software GeoGebra en el aprendizaje de la geometría en estudiantes de cuarto grado de secundaria. Estos diseños se sustentan en la formación de dos grupos de personas o estudiantes con característica no homogéneas, un 
grupo experimental y otro de control. Se elaboró una prueba de salida para ambos grupos. El esquema del diseño cuasi-experimental se muestra en la tabla 2.

Tabla 2. El diseño se diagrama de la siguiente manera

\begin{tabular}{|c|c|c|c|}
\hline Grupos & Pre test & Tratamiento & Test \\
\hline $\mathrm{GE}$ & & $x$ & $\mathrm{O} 1$ \\
\hline$G C$ & & $Y$ & $\mathrm{O} 2$ \\
\hline
\end{tabular}

Fuente: Metodología de Investigación. Hernández, Fernández y Baptista (2014).

Donde:

GE y GC: Grupo experimental y control

01: Medición del test en el grupo experimental

02: Medición del test en el grupo control

$X$ : Tratamiento con el uso del software educativo GeoGebra.

Y: Tratamiento sin el uso del software.

\section{POBLACIÓN Y MUESTRA}

La población de estudio estuvo conformada por 67 estudiantes de cuarto grado de la insitución educativa "Señor de los Milagros", distribuidos de la siguiente manera: 36 estudiantes en el grupo experimental y 31 en el grupo de control. Los alumnos ya estaban asignados por grupos o secciones previamente a la investigación, por lo que no se asignaron aleatoriamente y por lo tanto no son grupos equivalentes. La distribución de la muestra se observa en la tabla 3.

Tabla 3. La distribución de la muestra del estudio.

\begin{tabular}{ccc}
\hline \multicolumn{1}{c}{ Grupos } & GE & GC \\
\hline Estrategias & Con GeoGebra & Sin GeoGebra \\
Participantes & 36 & 31 \\
\hline Total & & 67 \\
\hline
\end{tabular}

Fuente: Elaboración propia. 


\section{TÉCNICAS E INSTRUMENTOS}

\section{TÉCNICAS}

La técnica consistió en el desarrollo de la sesión de clases en laboratorio de cómputo usando el software GeoGebra, dentro de la jornada de clases de la institución educativa. Los alumnos contaron con guías de aprendizaje, entregadas al iniciar. Por su parte, los alumnos del grupo de control no asistieron al laboratorio de cómputo, solo trabajaron con guías de aprendizaje elaboradas sin el uso del software GeoGebra, y se desarrollaron empleando la sesión de clases. En ambos grupos de estudio se empleó la técnica de test para medir el aprendizaje de la geometría en estudiantes de cuarto grado de secundaria de la institución educativa, "Señor de los Milagros". Por último, para la presentación, análisis e interpretación de resultados se utilizó la estadística descriptiva y la estadística inferencial.

\section{INSTRUMENTOS DE INVESTIGACIÓN}

Se elaboró una prueba de evaluación del aprendizaje en geometría, siendo el objetivo de la prueba medir la influencia del software GeoGebra en el aprendizaje de la geometría en estudiantes de cuarto grado de secundaria de la institución educativa. La prueba está compuesta por un total de 20 ítems para evaluar las tres dimensiones observadas: aprendizaje de las propiedades de los triángulos, aprendizaje de las propiedades de los cuadriláteros y el aprendizaje de las propiedades de la circunferencia. Cada pregunta correctamente contestada equivale a un punto, de tal manera que el puntaje máximo es de 20. La distribución de los ítems de la prueba aparece en la tabla 4

Tabla 4. Especificaciones de la prueba para medir la variable dependiente

\begin{tabular}{llcc}
\hline \multicolumn{1}{c}{ Dimensión } & \multicolumn{1}{c}{$\begin{array}{c}\text { Estructura de la prueba de post test } \\
\text { Ítems }\end{array}$} & Total & Porcentaje \\
\hline Propiedades del triángulo & $1,2,3,4,5,6,7$ & 7 & 35 \\
Propiedades del cuadrilátero & $8,9,10,11,12,13,14$ & 7 & 35 \\
Propiedades de la circunferencia & $15,16,17,18,19,20$ & 6 & 30 \\
\hline \multicolumn{2}{c}{ Total de ítems } & 20 & 100 \\
\hline
\end{tabular}

Fuente: Elaboración propia. 
La prueba de evaluación se validó por cinco expertos, cuyo resultado de Alfa de Cronbach tiene un valor de $\alpha=0.845$ que corresponde a una escala de excelente de validez. Para determinar el grado de confiabilidad de la prueba, se eligió una muestra piloto de 10 estudiantes de similar característica al grupo de intervención, a los cuales se les aplicó una prueba. A partir de los datos obtenidos, se estimó el coeficiente de confiabilidad de la prueba, por el método de dos mitades. Posteriormente, se estableció el nivel de correlación existente entre los puntajes obtenidos en ambas mitades. Para lo cual se utilizó el coeficiente de correlación de Pearson (r). Así tenemos:

$$
r=\frac{n \sum X Y-\sum X \sum Y}{\sqrt{\left[n \sum X^{2}-\left(\sum X\right)^{2}\left[n \sum Y^{2}-\left(\sum Y\right)^{2}\right]\right.}}
$$

Donde:

$n$ : Es el número de alumnos evaluados de la muestra piloto.

$X$ : Puntaje obtenido en las preguntas pares.

Y: Puntaje obtenido en las preguntas impares.

Finalmente se hace el ajuste a través del coeficiente de Spearman-Brow, lo cual nos permite determinar la confiabilidad de la prueba completa, así tenemos:

$$
r_{s}=\frac{2 r_{x y}}{1+r_{x y}}
$$

Donde:

$r_{s}$ : Confiabilidad estimada para la prueba completa.

$r_{x y}$ : Correlación de Pearson entre las dos mitades (r). 
Tabla 5. Grado de confiablidad de la prueba para medir el aprendizaje de la geometría.

\begin{tabular}{|l|l|l|c|}
\hline \multirow{3}{*}{ Alfa de Cronbach } & \multirow{2}{*}{ Parte 1 } & Valor & 0.623 \\
\cline { 2 - 4 } & & $N^{\circ}$ de elementos & $10^{\mathrm{a}}$ \\
\cline { 2 - 4 } & \multirow{2}{*}{ Parte 2 } & Valor & 0.78 \\
\cline { 2 - 3 } & & $N^{\circ}$ de elementos & $10^{\mathrm{b}}$ \\
\cline { 2 - 3 } & $N^{\circ}$ total de elementos & 20 \\
\hline \multicolumn{2}{|l|}{ Correlación entre formularios } & Longitud igual & 0.704 \\
\hline \multirow{2}{*}{ Coeficiente de Spearman-Brown } & 0.826 \\
\cline { 2 - 3 } & Longitud desigual & 0.826 \\
\hline \multicolumn{2}{|l|}{ Coeficiente de dos mitades de Guttman } & 0.817 \\
\hline
\end{tabular}

Fuente: Elaboración propia.

De la tabla 5 se obtuvo el coeficiente de dos mitades de Guttman 0.817, que corresponde a una escala excelente de confiabilidad.

\section{MÉTODOS}

La evaluación de las tres dimensiones empleó los datos obtenidos de la prueba de evaluación del aprendizaje en geometría, que se aplicó a los grupos experimental y de control, antes y después de la intervención del programa experimental. La aplicación de la prueba antes de intervención del programa experimental corroboró la equivalencia del desempeño de ambos grupos en las capacidades evaluadas. Luego vino la intervención, con 12 sesiones de clases de dos horas cada una, que se desarrolló en dos meses. Posteriormente, la aplicación después de la intervención estableció las diferencias significativas en el aprendizaje de la geometría de los estudiantes del grupo experimental en comparación con el grupo de control. Para el análisis de la investigación se utilizó la estadística descriptiva y para la prueba de hipótesis, se hizo la prueba de hipótesis de medias de dos poblaciones normales independientes de varianzas desconocidas, debido a que la intervención se realizó con dos grupos. 


\section{RESULTADOS}

\section{A) ANÁLISIS DESCRIPTIVO}

\section{ANÁLISIS DESCRIPTIVO DEL APRENDIZAJE DE LA GEOMETRÍA}

En la tabla 6 se muestra el promedio aritmético de las notas de los estudiantes que conformaron el grupo experimental y el grupo de control, siendo el promedio aritmético 13.3611 y 8.9354 puntos respectivamente. La diferencia de las notas de los alumnos del grupo experimental y del grupo de control es de 4.4257 puntos a favor de los alumnos que conformaron el grupo experimental. Demostrándose de esta forma que con el empleo del software GeoGebra, base de la estrategia didáctica utilizada en 12 sesiones de clase, mejoró significativamente el aprendizaje de la geometría en estudiantes de cuarto grado de secundaria.

Tabla 6. La estadística de las notas de los alumnos del grupo experimental y del grupo de control.

\begin{tabular}{lcc}
\hline & $\begin{array}{c}\text { Resultados estadísticos del } \\
\text { grupo experimental }\end{array}$ & $\begin{array}{c}\text { Resultados estadísticos del grupo } \\
\text { de control }\end{array}$ \\
\hline Media & 13.3611 & 8.9354 \\
Error típico & 0.4994 & 0.5992 \\
Moda & 12 & 5 \\
Desviación estándar & 2.9966 & 3.3360 \\
Varianza de la muestra & 8.9801 & 11.1290 \\
Coeficiente de asimetría & 0.2775 & 0.2271 \\
\hline Estudiantes & 36 & 31 \\
\hline
\end{tabular}

Fuente: Procesamiento de datos obtenidos de la investigación.

La representación gráfica de las notas de los alumnos del grupo experimental y del grupo de control de la tabla 6, aparecen en la figura 1. 


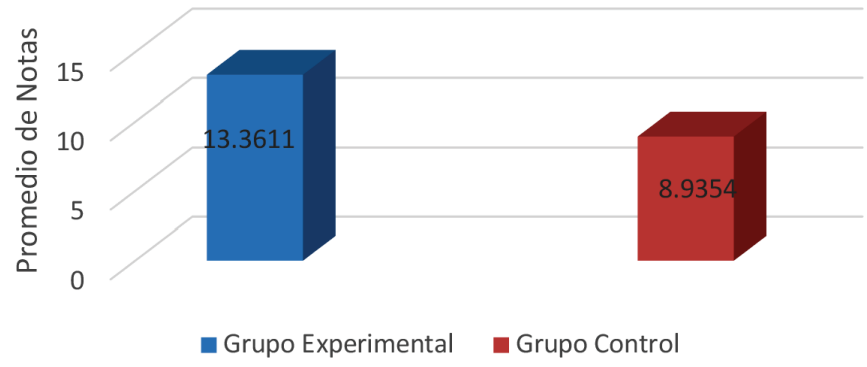

Figura 1. Comparación de las notas de los alumnos del grupo experimental y del grupo de control.

\section{ANÁLISIS DESCRIPTIVO DEL APRENDIZAJE DE LAS PROPIEDADES DE LOS TRIÁNGULOS}

En la tabla 7, se muestra el promedio aritmético de las notas de los estudiantes de la dimensión del aprendizaje de las propiedades de los triángulos, siendo 4.6111 y 3.2581 puntos respectivamente de un total de siete puntos. La diferencia de notas de los alumnos del grupo experimental y del grupo de control es de 1.3530 puntos a favor de los alumnos que conformaron el grupo experimental. Esto significa que el empleo del software GeoGebra como base de la estrategia didáctica, mejoró significativamente en el aprendizaje de las propiedades de los triángulos en estudiantes de cuarto grado de secundaria.

Tabla 7. La estadística de las notas de los alumnos en el aprendizaje de las propiedades de los triángulos.

\begin{tabular}{lcc}
\hline & $\begin{array}{c}\text { Resultados estadísticos } \\
\text { del grupo experimental }\end{array}$ & $\begin{array}{c}\text { Resultados estadísticos del } \\
\text { grupo de control }\end{array}$ \\
\hline Media & 4.6111 & 3.2581 \\
Error típico & 0.2042 & 0.2072 \\
Moda & 4 & 3 \\
Desviación estándar & 1.2254 & 1.1538 \\
Varianza de la muestra & 1.5016 & 1.3312 \\
Coeficiente de asimetría & 0.3195 & 0.4303 \\
\hline Estudiantes & 36 & 31 \\
\hline
\end{tabular}

Fuente: Procesamiento de datos obtenidos de la investigación, 2019. 
La representación gráfica de las notas de los alumnos en el aprendizaje de las propiedades de los triángulos de la tabla 7, aparece en la figura 2.

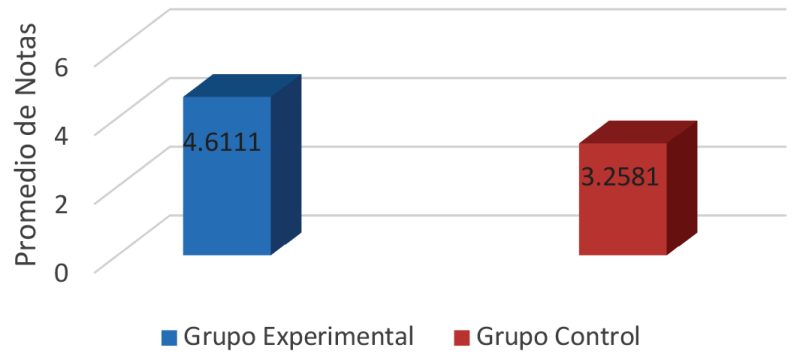

Figura 2. Comparación de las notas de los alumnos del aprendizaje de las propiedades de los triángulos.

\section{ANÁLISIS DESCRIPTIVO DEL APRENDIZAJE DE LAS PROPIEDADES DE LOS CUADRILÁTEROS}

En la tabla 8, se muestra el promedio aritmético de las notas de los estudiantes en el aprendizaje de las propiedades de los cuadriláteros y es de 4.6111 y 3.0968 puntos respectivamente de un total de siete puntos. La diferencia de notas de los alumnos del grupo experimental y del grupo de control es de 1.5143 puntos, también a favor de los alumnos que conformaron el grupo experimental. Esto significa que, el empleo del software GeoGebra como base de la estrategia didáctica, mejoró significativamente en el aprendizaje de las propiedades de los cuadriláteros en estudiantes del cuarto grado de secundaria. 
Tabla 8. La estadística de las notas de los alumnos en el aprendizaje de las propiedades de los cuadriláteros.

\begin{tabular}{lcc}
\hline & $\begin{array}{c}\text { Resultados estadísticos del } \\
\text { grupo experimental }\end{array}$ & $\begin{array}{c}\text { Resultados estadísticos } \\
\text { del grupo de control }\end{array}$ \\
\hline Media & 4.6111 & 3.0968 \\
Error típico & 0.1838 & 0.2381 \\
Moda & 4 & 2 \\
Desviación estándar & 1.1027 & 1.3255 \\
Varianza de la muestra & 1.2159 & 1.7569 \\
Coeficiente de asimetría & -0.0956 & 0.2703 \\
\hline Estudiantes & 36 & 31 \\
\hline
\end{tabular}

Fuente: Procesamiento de datos obtenidos de la investigación, 2019.

La representación gráfica de las notas de los alumnos en el aprendizaje de las propiedades de los cuadriláteros de la tabla 8, aparece en la figura 3.

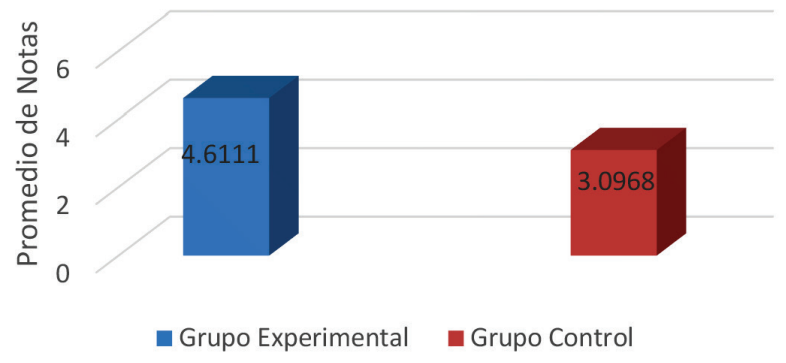

Figura 3. Comparación de las notas de los alumnos en el aprendizaje de las propiedades de los cuadriláteros.

\section{ANÁLISIS DESCRIPTIVO DEL APRENDIZAJE DE LAS PROPIEDADES DE LA CIRCUNFERENCIA}

En la tabla 9 se muestra el promedio aritmético de las notas de los alumnos del aprendizaje de las propiedades de la circunferencia, siendo esto 4.1111 y 2.4516 puntos respectivamente de un total de seis puntos. La diferencia de notas de los alumnos del grupo experimental y del grupo de control es de 1.6595 puntos a favor de los alumnos que conformaron el grupo experimental. Esto significa que, el empleo del software GeoGebra como base de la estrategia didáctica, mejoró 
significativamente el aprendizaje de las propiedades de la circunferencia en estudiantes de cuarto grado de secundaria.

Tabla 9. Estadístico de las notas de los alumnos en el aprendizaje de las propiedades de la circunferencia.

\begin{tabular}{lrc}
\hline & $\begin{array}{c}\text { Resultados estadísticos del grupo } \\
\text { experimental }\end{array}$ & $\begin{array}{c}\text { Resultados estadísticos del } \\
\text { grupo de control }\end{array}$ \\
\hline Media & 4.1111 & 2.4516 \\
Error típico & 0.2174 & 0.2167 \\
Moda & 5 & 3 \\
Desviación estándar & 1.3044 & 1.2066 \\
Varianza de la muestra & 1.7016 & 1.4559 \\
Coeficiente de asimetría & 0.1104 & 0.0006 \\
\hline Estudiantes & 36 & 31 \\
\hline
\end{tabular}

Fuente: Procesamiento de datos obtenidos de la investigación, 2019.

La representación gráfica de las notas de los alumnos en el aprendizaje de las propiedades de la circunferencia de la tabla 9, aparece en la figura 4.

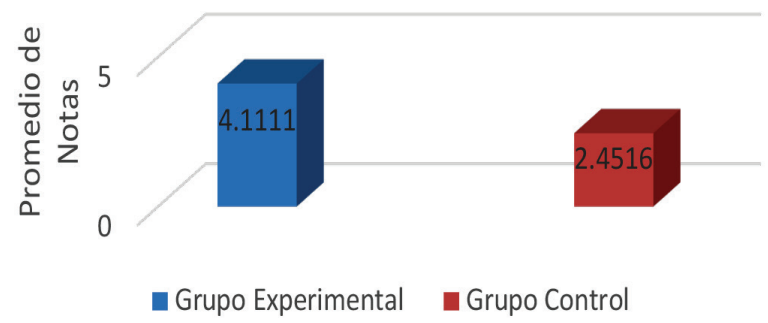

Figura 4. Comparación de las notas de los alumnos del aprendizaje de las propiedades de la circunferencia. 


\section{B) ANÁLISIS INFERENCIAL}

\section{PRUEBA DE LA HIPÓTESIS GENERAL}

Para hacer la prueba de hipótesis, se utilizó la prueba de hipótesis de medias de dos poblaciones normales independientes de varianzas desconocidas, además se utilizó el Teorema de Límite Central (Córdova, 2008).

i) Formulación de hipótesis nula y alternativa:

$\mathrm{H}_{0}: \mu_{1} \leq \mu_{2}$, el empleo del software GeoGebra no mejora significativamente en el aprendizaje de la geometría en estudiantes de cuarto grado de secundaria.

$\mathrm{H} 1: \mu_{1}>\mu_{2}$, el empleo del software GeoGebra mejora significativamente el aprendizaje de la geometría en estudiantes de cuarto grado de secundaria.

Donde $\mu_{1}$ y $\mu_{2}$, es el promedio poblacional de las notas del aprendizaje de la geometría de los estudiantes del grupo experimental y del grupo de control respectivamente.

ii) Establecer el nivel de significancia $(\alpha)$. Se considera $\alpha=0.05$

iii) Seleccionar el valor estadístico de prueba. En este caso la estadística de la prueba es la variable Z normal estandarizado dada por:

$$
\begin{aligned}
& Z_{\text {Cal }}=\frac{\left(\bar{X}_{1}-\bar{X}_{2}\right)}{\sqrt{\frac{s_{1}^{2}}{n_{1}}+\frac{s_{2}^{2}}{n_{2}}} \sim N(0,1)} \\
& Z_{\text {Cal }}=\frac{(13.3611-8.9354}{\sqrt{\frac{8.980158}{36}+\frac{11.12903}{31}}}=5.674
\end{aligned}
$$

iv) Establecer la región de rechazo y de la región de aceptación. Para determinar la región de rechazo y de aceptación de la hipótesis $\mathrm{H}_{0}$ se debe hacer la gráfica de la distribución normal estandarizada con un nivel de significancia de 0.05, dicha gráfica se muestra en la figura 5 : 


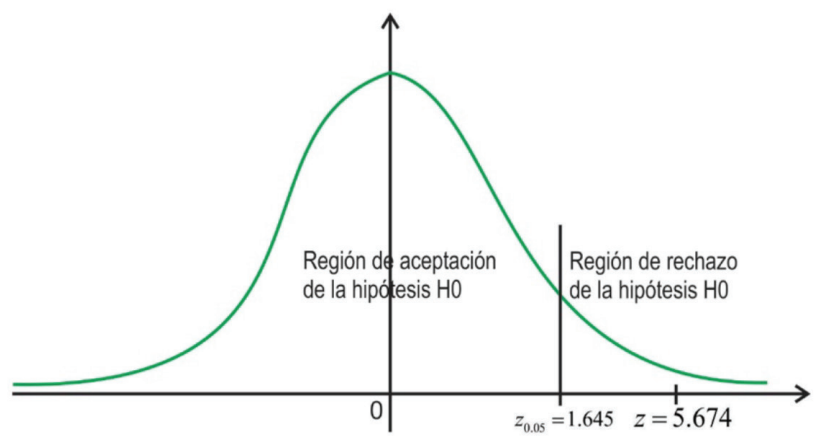

Figura 5. Región de rechazo y de aceptación de la hipótesis nula con un nivel de significancia $\alpha=0.05$

v) Tomar la decisión. De la figura 5 se observa que $\boldsymbol{z}_{\text {Cal }}=5.674>z_{0.05}=1.645$, es decir z calculado se encuentra dentro de la región de rechazo de la hipótesis $\mathrm{H}_{0}$, por lo tanto se rechaza la hipótesis $\mathrm{H}_{0}$ y se acepta la hipótesis H1, llegando a la conclusión que, el empleo del software GeoGebra mejora significativamente el aprendizaje de la geometría en estudiantes de cuarto grado de secundaria.

\section{PRUEBA DE LA HIPÓTESIS ESPECÍFICO 1}

i) Formulación de hipótesis nula y alternativa:

$\mathrm{H}_{0}: \mu_{1} \leq \mu_{2}$, el empleo del software GeoGebra no mejora significativamente en el aprendizaje de las propiedades de los triángulos en estudiantes de cuarto grado de secundaria.

H1: $\mu_{1}>\mu_{2}$, el empleo del software GeoGebra mejora significativamente en el aprendizaje de las propiedades de los triángulos en estudiantes de cuarto grado de secundaria.

Donde $\mu_{1}$ y $\mu_{2}$, es el promedio poblacional de las notas del aprendizaje de las propiedades de los cuadriláteros de los estudiantes del grupo experimental y del grupo de control respectivamente

ii) Establecer el nivel de significancia ( $\alpha$ ). Se considera $\alpha=0.05$.

iii) Seleccionar el valor estadístico de prueba. En este caso la estadística de la prueba es la variable Z normal estandarizado dada por: 


$$
\begin{aligned}
Z_{\text {Cal }} & =\frac{\left(\bar{X}_{1}-\bar{X}_{2}\right)}{\sqrt{\frac{s_{1}^{2}}{n_{1}}+\frac{s_{2}^{2}}{n_{2}}}} \sim N(0,1) \\
Z_{\text {Cal }} & =\frac{(4.6111-3.2581}{\sqrt{\frac{1.5016}{36}+\frac{1.3312}{31}}}=4.650
\end{aligned}
$$

iv) Establecer la región de rechazo y la región de aceptación. Para determinar la región de rechazo y de la región de aceptación de la hipótesis $\mathrm{H}_{0}$ se debe hacer la gráfica de la distribución normal estandarizada con un nivel de significancia de 0.05, dicha gráfica se muestra en la figura 6

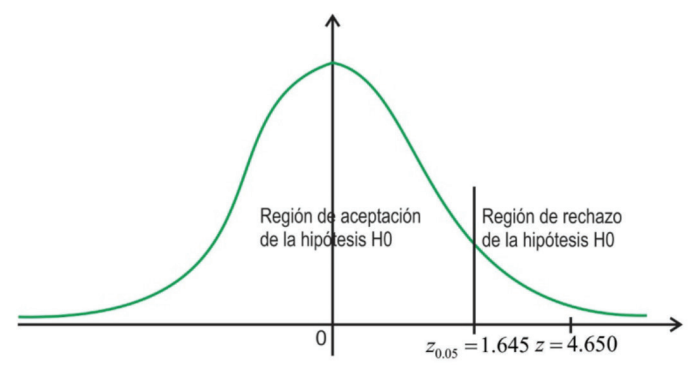

Figura 6. Región de rechazo y de aceptación de la hipótesis nula con un nivel de significancia $\alpha=0.05$

v) Tomar la decisión. De la figura 6 se observa que $z_{\text {Cal }}=4.650>z_{0.05}=1.645$, es decir z calculado se encuentra dentro de la región de rechazo de la hipótesis $\mathrm{H}_{0}$, por lo tanto se rechaza la $\mathrm{H}_{0}$ y se acepta la hipótesis $\mathrm{H} 1$, llegando a la conclusión que, el empleo del software GeoGebra mejora significativamente el aprendizaje de las propiedades de los triángulos en estudiantes de cuarto grado de secundaria.

\section{PRUEBA DE HIPÓTESIS DE LA HIPÓTESIS ESPECÍFICO 2}

i) Formulación de hipótesis nula y alternativa:

$\mathrm{H}_{0}: \mu_{1} \leq \mu_{2}$, el empleo del software GeoGebra no mejora significativamente en el aprendizaje de las propiedades de los cuadriláteros en estudiantes de cuarto grado de secundaria. 
$\mathrm{H} 1: \mu_{1}>\mu_{2}$, el empleo del software GeoGebra mejora significativamente en el aprendizaje de las propiedades de los cuadriláteros en estudiantes de cuarto grado de secundaria.

Donde $\mu_{1}$ y $\mu_{2}$, es el promedio poblacional de las notas del aprendizaje de las propiedades de los cuadriláteros de los estudiantes del grupo experimental y del grupo de control respectivamente

ii) Establecer el nivel de significancia $(\alpha)$. Se considera $\alpha=0.05$.

iii) Seleccionar el valor estadístico de prueba. En este caso la estadística de la prueba es la variable Z normal estandarizado dada por:

$$
\begin{aligned}
Z_{\text {Cal }} & =\frac{\left(\bar{X}_{1}-\bar{X}_{2}\right)}{\sqrt{\frac{s_{1}^{2}}{n_{1}}+\frac{s_{2}^{2}}{n_{2}}}} \sim N(0,1) \\
Z_{\text {Cal }} & =\frac{(4.6111-3.0966)}{\sqrt{\frac{1.2154}{36}+\frac{1.7569}{31}}}=5.035
\end{aligned}
$$

iv) Establecer la región de rechazo y de la región aceptación. Para determinar la región de rechazo y de aceptación de la hipótesis $\mathrm{H}_{0}$ se debe hacer la gráfica de la distribución normal estandarizada con un nivel de significancia de 0.05, dicha gráfica se muestra en la figura 7 .

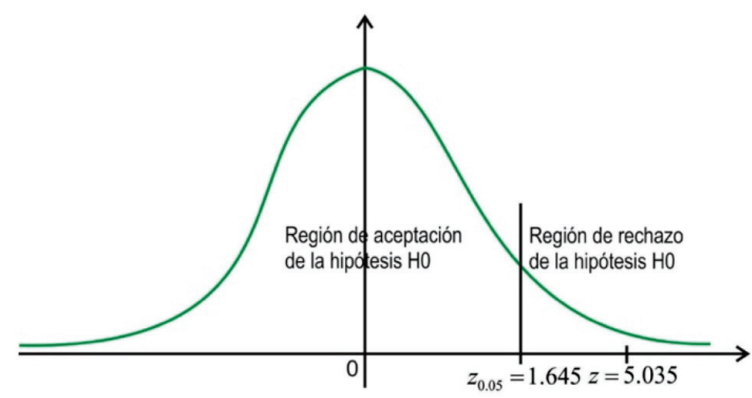

Figura 7. Región de rechazo y de aceptación de la hipótesis nula con un nivel de significancia $\alpha=0.05$ 
v) Tomar la decisión. De la figura 7 se observa que $\boldsymbol{z}_{\text {Cal }}=5.035>z_{0.05}=1.645$, es decir z calculado se encuentra dentro de la región de rechazo de la hipótesis $\mathrm{H}_{0}$, por lo tanto se rechaza la $\mathrm{H}_{0}$ y se acepta la hipótesis $\mathrm{H} 1$, llegando a la conclusión que, el empleo del software GeoGebra mejora significativamente el aprendizaje de las propiedades de los cuadriláteros en estudiantes de cuarto grado de secundaria.

\section{Prueba de hipótesis de la hipótesis específico 3}

i) Formulación de hipótesis nula y alternativa:

$\mathrm{H}_{0}: \mu_{1} \leq \mu_{2}$, el empleo del software GeoGebra no mejora significativamente en el aprendizaje de las propiedades de la circunferencia en estudiantes de cuarto grado de secundaria.

$\mathrm{H} 1: \mu_{1}>\mu_{2}$, el empleo del software GeoGebra mejora significativamente en el aprendizaje de las propiedades de la circunferencia en estudiantes de cuarto grado de secundaria.

Donde $\mu_{1}$ y $\mu_{2}$, es el promedio poblacional de las notas del aprendizaje de las propiedades de la circunferencia de los estudiantes del grupo experimental y del grupo de control respectivamente.

ii) Establecer el nivel de significancia ( $\alpha$ ). Se considera $\alpha=0.05$.

iii) Seleccionar el valor estadístico de prueba. En este caso la estadística de la prueba es la variable Z estandarizado dada por:

$$
\begin{aligned}
Z_{\text {Cal }}= & \frac{\left(\bar{X}_{1}-\bar{X}_{2}\right)}{\sqrt{\frac{s_{1}^{2}}{n_{1}}+\frac{s_{2}^{2}}{n_{2}}}} \sim N(0,1) \\
Z_{\text {Cal }} & =\frac{(4.1111-2.4515)}{\sqrt{\frac{1.7016}{36}+\frac{1.4559}{31}}}=5.406
\end{aligned}
$$

iv) Establecer la región de rechazo y de la región de aceptación. Para determinar la región de rechazo y de aceptación de la hipótesis $\mathrm{H}_{0}$ se debe hacer la gráfica de la distribución normal z estandarizada con un nivel de significancia de 0.05, dicha gráfica se muestra en la figura 8: 


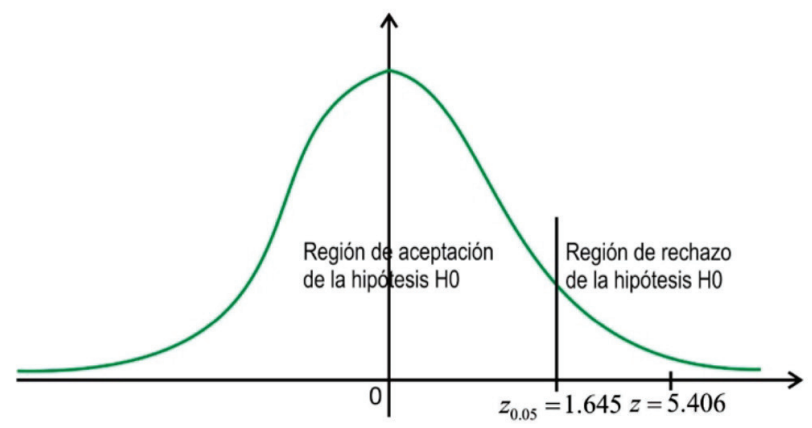

Figura 8. Región de rechazo y de aceptación de la hipótesis nula con un nivel de significancia $\alpha=0.05$

v) Tomar la decisión. De la figura 8 se observa que $z_{\text {Cal }}=5.406>z_{0.05}=1.645$, es decir z calculado se encuentra dentro de la región de rechazo de la hipótesis $\mathrm{H}_{0}$, por lo tanto se rechaza la $\mathrm{H}_{0}$ y se acepta la hipótesis $\mathrm{H} 1$, llegando a la conclusión que, el empleo del software GeoGebra mejora significativamente el aprendizaje de las propiedades de la circunferencia en estudiantes de cuarto grado de secundaria.

\section{SÍNTESIS DE RESULTADOS}

Los resultados del trabajo de investigación demuestran que, el uso del software GeoGebra como base de la estrategia didáctica en sesiones de aprendizaje, mejoró significativamente en el aprendizaje de la geometría en estudiantes de cuarto grado de secundaria. Los estudiantes del grupo experimental y del grupo de control obtuvieron una nota en promedio aritmético de 13.3611 y 8.9354 puntos respectivamente. Lo que coincide con la investigación de Díaz-Nunja (2017). En relación con el aprendizaje de las propiedades de los triángulos, se observó, que el uso del software GeoGebra mejoró significativamente en el aprendizaje de las propiedades de los triángulos y que en una prueba de 7 puntos, los estudiantes del grupo experimental y del grupo de control obtuvieron una nota en promedio de 4.6111 y 3.2581 puntos respectivamente. Esto confirma los resultados encontrados por Ruiz (2012). En cuanto al aprendizaje de las propiedades de los cuadriláteros se observó, que en una prueba de 7 puntos, los estudiantes del grupo experimental y del grupo de control obtuvieron una nota en promedio de 4.6111 y 3.0966 puntos respectivamente. Aquí encontramos 
una similitud importante con relación a los hallazgos encontrados por Pablo (2016), donde los estudiantes del grupo experimental obtuvieron un puntaje promedio de 14.50 puntos, a diferencia de los estudiantes del grupo de control que obtuvieron un puntaje promedio de 12.53 puntos. Por último, se observó que en el aprendizaje de las propiedades de la circunferencia, en una prueba de 6 puntos, los estudiantes del grupo experimental y control obtuvieron una nota en promedio de 4.1111 y 2.4515 puntos respectivamente.

\section{CONCLUSIONES}

Los resultados evidencian un comparación entre pruebas realizadas por estudiantes que siguieron una propuesta fundamentada en el uso de GeoGebra y un grupo de estudiantes que siguió una enseñanza tradicional, caracterizada por el uso de lápiz y papel. Un análisis sobre el tipo de tareas propuestas y la forma en que GeoGebra fue utilizado, permitiría mostrar las actividades de los estudiantes y la forma en que construyeron las propiedades de los triángulos, los cuadriláteros y la circunferencia. Es importante señalar, que los resultados cuantitativos, son considerados una evidencia sólida para dialogar con instancias decisivas en la innovación curricular y particularmente en la integración de GeoGebra -como ejemplo de software de geometría dinámica- para su inclusión en el corto, mediano y largo plazo en la enseñanza de la geometría. Asimismo, estos resultados sugieren la necesidad de crear talleres de capacitación basados en el uso de las tecnologías para los docentes de matemática, lo que permitiría asegurar la innovación didáctica constante, y atender una de las demandas pedagógicas y tecnológicas que impulsó el MINEDU. Esta formación especializada permitiría a los docentes, particularmente de las instituciones educativas de la región de Madre de Dios, incorporar en sus clases el uso del software GeoGebra. Lo que implicaría por supuesto, una reorganización didáctica estrechamente relacionada con la dimensión epistemológica de la geometría. Resulta necesario realizar más trabajos de investigación sobre el uso del software GeoGebra y la forma en que puede ser incorporado en las estrategias de enseñanza utilizadas por los docentes en la enseñanza de la geometría y en otras asignaturas matemáticas, que forman parte del programa curricular de Educación Secundaria. De manera general, el aporte de este estudio es mostrar la necesidad de incorporar al proceso de enseñanza de la geometría, el software GeoGebra, pues ofrece resultados en el aprendizaje de los estudiantes y sugiere al docente de matemática, que se trata de un 
recurso didáctico tecnológico con potencialidad para estudiar la geometría de manera dinámica, pero con el rigor matemático. Lo que permitiría, al menos inicialmente, re-equilibrar su uso con el de la pizarra física y el plumón utilizado en la enseñanza tradicional, que suele conducir a una forma mecánica de plantear los temas de triángulos, cuadriláteros y circunferencias. En cuanto al proceso de aprendizaje, al estudiante se le permitiría interactuar con los objetos y sus relaciones geométricas para adquirir conocimientos y aplicarlos a la resolución de problemas de la geometría.

\section{REFERENCIAS}

Almeida, M. (2002). Desarrollo Profesional Docente en Geometría: análisis de un proceso de Formación a Distancia. (Tesis de doctorado no publicada). Universidad Barcelona de España.

Bulut, M., y Bulut, N. (2011). Preservice teachers' usage of dynamic mathematics software. TOJET: The Turkish Online Journal of Educational Tecnology, 10(4), 294-299.

Cárdenas, G. E. (2018). Influencia del software educativo Winplot en el aprendizaje de las funciones cuadráticas en los estudiantes del cuarto grado de secundaria de la Institución Educativa № 1260. (Tesis de maestría no publicada). Universidad Nacional Mayor de San Marcos, Lima-Perú. https://cybertesis.unmsm.edu.pe/handle/20.500.12672/10112

Córdoba, F. J., Herrera, H. J., y Restrepo, C. M. (2013). Impacto del uso de objetos de aprendizaje en el desempeño en mamáticas de estudiantes del grado noveno. Revista Virtual de la Universidad Católica del Norte, 2(39), 47-58.

Córdova, M. (2008). Estadística Aplicada. MOSHERA S.R.L.

Díaz-Nunja, L. A. (2013). La influencia del software GeoGebra en el aprendizaje del álgebra de los alumnos del 4to año de secundaria de la Institución Educativa Trilce de la Molina, periodo 2021. (Tesis de maestría no publicada). Universidad de Educación Enrique Guzmán y Valle. https://repositorio.une.edu.pe/handle/UNE/438

Díaz-Nunja, J. A. (2017). La influencia del software GeoGebra en el aprendizaje del álgebra de los alumnos del 4 to año de educación secundaria de la Institución Educativa Trilce del Distrito de Santa Anita. (Tesis de maestría no publicada). Universidad Nacional de Educación Enrique Guzmán y Valle. https://repositorio.une.edu.pe/handle/UNE/1371 
Diaz-Nunja, L., Rodríguez-Sosa, J., y Lingán, K. S. (2018). Enseñanza de la geometría con el software GeoGebra en estudiantes secundarios de una institución educativa en Lima. Propósitos y Representaciones, 6(2), 217-234.

Duval, R. (2000). Basic issues for research in mathematics education. En. T. Nakahara y M. Koyama (Eds.), Proceedings of the 24th PME. International conference, 2 (pp. 55-69). PME.

Falen, R. (2017). Uso de software GeoGebra en el aprendizaje de la línea de Matemáticas Aplicadas II de la carrera de Computación e Informática en el Instituto de Educación Superior Público República Federal de Alemania Chiclayo. (Tesis de doctorado no publicada). Universidad Nacional de Educación Enrique Guzmán y Valle, Lima-Perú. https://repositorio.une.edu.pe/bitstream/handle/UNE/1693/TD\%20CE\%20 1681\%20F1\%20-\%20Falen\%20Larrea.pdf?sequence=1反isAllowed=y

Ferreira, N., Guerra Cardoso, H., Santos Macedo, E., Brevario, D., y Arnholdt- Schmitt, B. (2009). Trabajo Conjetural con el uso de GeoGebra (Ed.), Memorias del Sexto Congreso Internacional de Matemática Asistida por Computadora (CIEMAC 6)- Costa Rica.

Gamboa R., y Ballesteros, E. (2009). Algunas reflexiones sobre la didáctica de la geometría. Cuadernos de Investigación y Formación en Educación Matemática, 4(5), 113-136.

Gross-Salvat, B. (2000). El Ordinador invisible: hacia la apropiación del ordenador en la enseñanza. Gedisa.

Hernández, R., Fernández, C., y Batista, P. (2014). Metodología de la investigación (Sexta Edición ed.). McGraw-Hill.

Hohenwarter , J., Hohenwarter , M., y Lavicza, Z. (2009). Introducing dynamic mathematic software to secondary school teachers: The case of GeoGebra. Journal of Computers in Mathematics and Science Teaching, 28(2), 135-146.

Hohenwarter, M. (2002). GeoGebra-a software system for dynamic geometry and algebra in the plane. Unpublished Edition. Universidad de Salzburgo, Austria.

Jones, K. (2002). Issues in the teaching and learning of geometry. En L. Haggarty (Ed.), Aspects of Teaching Secundary Matemátics: perspectives on practice, (pp.121-139). Falmer Routledge.

Lamana-Selva, M. T., y De La Peña, C. (2018). Rendimiento Académico en Matemáticas. Revista Mexicana de Investigación Educativa, 23(79), 1076-1092.

López-Salmerón, N. (2006). El empleo de software Cabri Geometri Il en la enseñanza de la geometría en la Universidad Autónoma de Guerrero - México. Universidad Autónoma de Guerrero.

Mejía, E. (2005). Metodología de la investigación Científica. San Marcos. 
Mendes, T., Nascimento, G., Coelho Neto, J., Lucas, S., Pereira, R., y Bernadelli, M. (2014). The use of software GeoGebra as an Mathematics. Revista Espacios, 2-6.

MINEDU. (2017). Currículo Nacional de la Educación Básica del Miniesterio de Educación. Ministerio de Educación.

MINEDU. (2016). Programa Curricular de Educación Secundaria. Ministerio de Educación. Murillo Hernández, W. (2008). Investigación Científica.

Pedhazur, E. J., y Pedhazur Schmelkin, L. (1991). Medición, diseño y análisis: un enfoque integrado. Lawrence Erlbaum Associates.

Peréz-Gómez, A. (1988). Análisis didáctico de las teorías del aprendizaje. Universidad de Málaga.

Pablo, M. (2016). Influencia del software GeoGebra en el aprendizaje de la geometría análiitica en los estudiante de quinto grado de secundaria de la institución educativa Jose de la Torre Ugarte, El Augustino-2015. (Tesis de doctorado no publicada). Universidad Nacional de Educación Enrique Guzmán y Valle. https://repositorio.une. edu.pe/handle/UNE/2006

Ruiz-López, N. (2012). Análisis del desarrollo de competencias geométricas y didácticas mediante el software de geometría dinámica GeoGebra en la formación inicial del profesor de primaria. (Tesis de doctorado no publicada). Universidad Autónoma de Madrid.

Tatar, E., y Yilmaz, Z. (2016). Conceptual Understanding of Definite Integral with GeoGebra. Computers in the Schools. Intersdisciplinary Journal of Practice, Theory and Applied Research, 33(2), 120-132. http://doi.org/10.1080/07380569.2016.117480

Eliseo Pumacallahui Salcedo

Dirección: Tambopata, Puerto Maldonado

Teléfono: $\quad 95627745$ 